\title{
Anabases
}

ANABASES Traditions et réceptions de l'Antiquité

$21 \mid 2015$

Varia

\section{Peut-on traduire " savamment » tout en faisant des bévues incroyables ? Andreas Divus et son Aristophane}

Simone Beta

\section{(2) OpenEdition \\ Journals \\ Édition électronique \\ URL : http://journals.openedition.org/anabases/5287 \\ DOI : 10.4000/anabases.5287 \\ ISSN : 2256-9421 \\ Éditeur \\ E.R.A.S.M.E.}

\section{Édition imprimée}

Date de publication : 1 avril 2015

Pagination : 125-138

ISSN : $1774-4296$

Référence électronique

Simone Beta, « Peut-on traduire « savamment » tout en faisant des bévues incroyables ? Andreas Divus et son Aristophane», Anabases [En ligne], 21 | 2015, mis en ligne le 01 avril 2018, consulté le 21 octobre 2019. URL : http://journals.openedition.org/anabases/5287 ; DOI : 10.4000/anabases.5287 


\section{Peut-on traduire "savamment» tout en faisant des bévues incroyables? Andreas Divus et son Aristophane}

Simone BETA

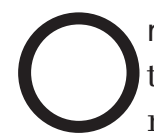

n sait que, à la Renaissance, les comédies d'Aristophane faisaient partie des textes qu'il fallait absolument lire et connaitre parce que le poète athénien était considéré un modèle parfait du dialecte attique. Dans une lettre envoyée à John Claymond, le premier président du collège Corpus Christi d'Oxford, Érasme de Rotterdam fait d'Aristophane un exemple de pureté stylistique à égalité avec Démosthène et Lucien; dans son traité sur la méthode pour étudier, lire et interpréter les auteurs, il inclut Aristophane dans son canon des meilleurs poètes, avec Homère et Euripide'.

C'est pour cette raison que, au début du xv siècle, bien avant que la première édition du texte grec d'Aristophane fût publié à Venise par l'imprimeur Aldo Manuzio, les premiers humanistes choisirent de traduire, à coté d'auteurs comme Platon et Aristote, Aristophane. Leonardo Bruni, l'humaniste arétin qui, après avoir suivi les leçons de Manuele Crisolora, traduisit les trois cent premiers vers du Ploutos, déclare dans la préface de sa traduction qu'il avait voulu montrer à ses contemporains quel était le genre de drames du poète comique: Ego igitur volens

1 Pour la première citation, voir l'epistula I66I (The correspondence of Erasmus: Letters I658-I8OI (I526-I527), Toronto and London, I974, vol. I2, p.I7); pour la deuxième, voir De ratione studii, éd. J.-C.Margolin, in Desiderii Erasmi Roterodami, Opera omnia I-2, Amsterdam, I97I, p. II5 (Quo quidem in genere (sc. dans la prose) primas tribuerim Luciano, alteras Demostheni, tertias Herodoto. Rursum ex poetis primas Aristophani, alteras Homero, tertias Euripidi). 
latinis ostendere quale genus erat illarum comediarum, primum actum huius comediae Aristophanis in latinum contuli ${ }^{2}$.

Et c'est également pour cette raison que, après les premières éditions du texte grec d'Aristophane, c'est-à-dire les neuf comédies publiées par Manuzio en I498, les deux autres comédies publiées par Bernardo Giunta à Florence en ı5ı6, la première édition vraiment intégrale des onze comédies due à Symon Grynaeus et publiée à Bâle chez l'éditeur Andreas Cratander en I532, un professeur de Capodistrie, Andreas Divus, publia à Venise, chez l'éditeur Giacomo Pocatela, la première traduction complète d'Aristophane ${ }^{3}$.

Bien que cette version, qui parut en i538, ne soit pas parfaite (et nous verrons bientôt pourquoi), le rôle qu'elle a joué dans la connaissance de l'œuvre d'Aristophane a été extrêmement important: cette traduction remporta un vif succès et fut réimprimée six fois entre 1539 et 1597 par plusieurs imprimeurs ${ }^{4}$.

Dans sa préface, l'épître dédicatoire à Alvise Pisani, évêque de Padoue, Divus déclare n'avoir pas essayé de reproduire l'élégance, la beauté et le charme des mots: il s'est contenté de tracer les contours des mots (lineamenta) de la façon la plus fidèle possible, pour exprimer les figures poétiques du style d'Aristophane ${ }^{5}$. Or c'est précisément dans ce choix de traduire seulement les lineamenta des mots que se trouvent les mérites et les défauts de son entreprise: on peut parler de mérites, parce que sa traduction mot à mot a permis à qui possédait une connaissance très limitée de la langue grecque de comprendre une bonne partie du texte

2 La traduction de Bruni a été publiée par Maria et Enzo Cecchini (L. Bruni, Versione del Pluto di Aristofane, Firenze, ig65); voir aussi E. Cecchins et A.C. CAssio, «Due contributi alla traduzione di Leonardo Bruni del Pluto di Aristofane », Giornale italiano di filologia classica 24 (I972), p.472-82, et L.Pradelle, “La version latine du Ploutos d'Aristophane par Leonardo Bruni: une traduction expérimentale? », à paraître.

3 Aristophanis, comicorum principis, Comoediae undecim, e Graeco in Latinum, ad verbum translatae, Andrea Divo Iustinopolitano interprete, Venetijs, apud D. Iacob a Burgofrancho Papiensem, mense Iunio, i538.

4 Voici la liste de ces réimpressions: Andreas Cratander, Bâle, I539 et i542; Pocatela, Venise, I542; “ apud Cominum de Tridino Montisferrati », Venise, I548; Cratander, Bâle, I552; “apud I. Ba. Et I. Be. Sessam », Venise, ı597. Une copie de l'édition Bâle ı539 fut possédée par François Rabelais; L.C.Stevens, «Rabelais and Aristophanes », Studies in Philology55 (I958), p. 24-3o, nous dit que ce livre est maintenant dans la bibliothèque du Musée Condé, à Chantilly (cote VIII-E-oor).

5 Quapropter cum non ignorarem, quantum ex literis (sic!) Graecis fructus percipi soleat, nec tamen ab omnibus aut illis operam dari posse, aut intelligi: non inutilem, neque ingratum quam plurimis fore putavi laborem meus, si Graecos autores ad verbum in Latinum sermonem vertendos, atque excudendos curarem: non tamen in iis tam verborum quidem elegantiam, venustatem, lenociniave captans, quam ut lineamenta fidelius quam possem, poeticasque figuras exprimerem. 
d'Aristophane; mais on peut aussi parler de défauts, parce que le désir d'être toujours fidèle au texte original a produit une traduction qui est souvent incompréhensible, et très souvent incorrecte.

Les “savants » qui se sont intéressés à cette traduction «savante» ont toujours négligé ses mérites et souligné ses défauts. Célèbre est le jugement tranchant de Tanneguy Le Fèvre, qui en I674 traduisit en latin l'Assemblée des femmes: après avoir accusé Divus d'être imperitissimus et infantissimus, Tanneguy écrivit que le traducteur avait peiné à comprendre deux vers consécutifs (vix alternos versus Comici nostri intellexit) parce que la sensibilité pour la langue grecque lui faisait complètement défaut (qui nullum Hellenismi sensum haberet) ${ }^{6}$.

Dans un article paru en 2012 je me suis intéressée à sa traduction de la Lysistrata en soulignant, à côté de ses défauts, ses mérites de traducteur; dans cette contribution je tenterai d'en faire autant au sujet d'une autre comédie, qui permet d'analyser le langage particulier de la politique' . Il s'agit des Cavaliers, la comédie qu'Aristophane écrivit en 424 dans le but de convaincre ses concitoyens de ne pas voter pour Cléon, le démagogue qui avait pris la place de Périclès à la tête du parti démocratique.

6 Tanaquilli Fabri Epistolae, pars posterior, editio altera priori emendatior. Additae sunt

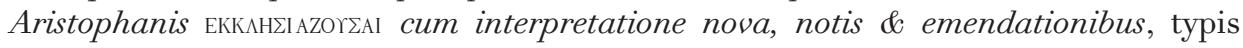
\& sumptibus Isaaci Desbordes \& Ioannis Lesnerii, Salmurii, i674. Pour une autre critique également sévère de la traduction de Divus on peut lire l'avant-propos de Ludolph Küster à l'édition complète des comédies d'Aristophane qu'il publia en ı Amsterdam, où le philologue allemand déclare qu'il n'a pas voulu utiliser ses versions parce qu'elles étaient pleines d'erreurs (Aristophanis Comoediae undecim, Graece et Latine, ex codd. mss. emendatae: cum scholiis antiquis, inter quae scholia in Lysistratam ex cod. Vossiano nunc primum in lucem prodeunt. Accedunt notae virorum doctorum in omnes comoedias; inter quas nunc primum eduntur Isaaci Casauboni in Equites; illustriss. Ezech. Spanhemii in tres priores; et Richardi Bentleji in duas priores comoedias observationes. Omnia collegit et recensuit, notasque in novem comoedias, et quatuor indices in fine adjecit Ludolphus Kusterus, sumptibus Thomae Fritsch, Amstelodami); voir aussi le jugement de l'abbé Giuseppe Fabiani dans Il Pluto di Aristofane, commedia prima, greco-italiana in versi con sue annotazioni, opera del signor Gio. Batista Terucci, gentiluomo sanese, pubblico professore di Leggi Civili nell'università di Siena, e accademico intronato, illustrata e pubblicata dall'abate Giuseppe Fabiani, Stamperia Moückiana, Firenze, I75r.

7 S. Beta, “La prima traduzione latina della Lisistrata. Luci e ombre della versione di Andrea Divo », Quaderni Urbinati di Cultura Classica Ioo (2012 (vol. I29), p. 95-114. Sur le langage particulier de la philosophie, voir J.NAssichuk, «Strepsiades’ Latin voice: two Renaissance translations of Aristophanes' Clouds », in S. Douglas Olson (ed.), Ancient Comedy and Reception. Essays in Honor of Jeffrey Henderson, Berlin and Boston, 2013, p. $427-446$. 
Au début de la pièce, on voit deux esclaves, deux serviteurs de Démos Pyknites, la personnification du peuple athénien, qui se réunissait sur la Pnyx; ils se plaignent de leur malheureuse condition depuis qu'un nouvel esclave, le Paphlagonien (la caricature de Cléon), est entré dans la maison de Démos.

Le premier esclave, qui avait sur le visage un masque avec les traits du stratège Démosthène, sort de la maison de Démos en s'écriant:

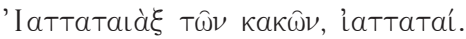

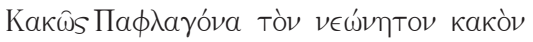

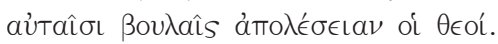

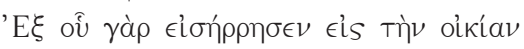

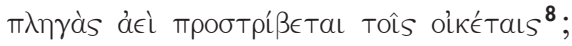

le deuxième esclave, qui avait sur le visage une masque représentant les traits du stratège Nicias, est d'accord avec les mots de son collègue:

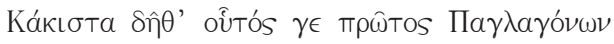

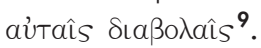

On peut voir très aisément comment la traduction de Divus, en suivant servilement la disposition du texte grec, vers à vers, mot à mot, pose parfois des problèmes:

Démosthène: Heu malis heu.

Male Paphlagona nuper emptum malum,

ipsis consiliis perdant Dei.

Ex quo enim ingressus est in domum,

plagas semper intulit famulis.

Nicias : Pessime certe, hic primus Paphlagonum

ipsis consiliis.

Si la traduction des paroles de Démosthène en latin est à peu près compréhensible, celle des paroles de Nicias nous laisse perplexes, parce que la phrase Pessime certe, hic primus Paphlagonum ipsis consiliis ne signifie pas que l'esclave a voulu

8 Aristophane, Cavaliers I-5: “Aïe! Aïe! Aïe! Hi! Malheur de malheur! Aïe! Aïe! Aie! Maudit soit le Paphlagonien acheté récemment, le misérable! Depuis que la malheur a voulu qu'il entrât dans la maison, il ne cesse de faire donner la raclée aux serviteurs ». Ici et dans les notes suivantes la traduction française est celle d'Hilaire van Daele, publiée en regard du texte grec édité par Victor Coulon dans la collection Budé, paru à partir de 1923.

9 Aristophane, Cavaliers 6-7: «Qu'il périsse le plus misérablement du monde, avant tous les autres Paphlagoniens, avec ses diffamations!». 
maudire le Paphlagonien. En sus, il faut souligner que Divus a traduit par le même

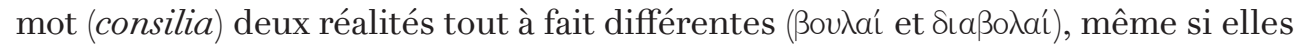
partagent la même racine, témoignant ainsi qu'il n'avait pas vraiment compris le sens du texte d'Aristophane - et confirmant par conséquent la critique sévère de Tanneguy Le Fèvre.

La recherche d'un moyen d'échapper au Paphlagonien emmène les deux esclaves à recourir aux vers d'Euripide: Nicias cite un vers du poète tragique (П⿵人s

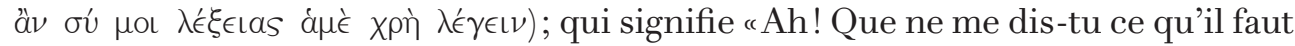
que je dise? »; Démosthène gronde son collègue, en disant:

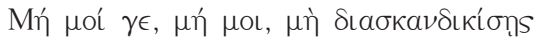

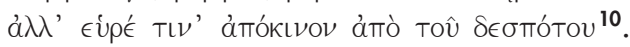

On peut aisément imaginer l'embarras de Divus devant cette réplique, puisque

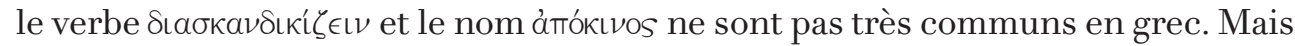
le texte qu'il avait utilisé avait un atout: le volume d'Aldo Manuzio, édité par le philologue crétois Marcus Musurus, était pourvu de scholies. Et les commentaires anciens nous donnent deux explications très importantes à ce sujet: d'abord, il disent que dans $\delta\llcorner a \sigma \kappa a \nu \delta \kappa \kappa i \zeta \epsilon \iota \nu$ il y avait le mot $\sigma \kappa a ́ \nu \delta\llcorner\xi$ (“ une herbe sauvage », c'està-dire une allusion méchante au métier de la mère d'Euripide, qui, pour les poète comiques, était une marchande de légumes); ensuite, il disent que l'àmókıvos était une danse très vive.

Devant ces difficultés réelles, il faut avouer que la traduction de Divus est assez créative :

Non mihi, non mihi ne olorizes.

Sed inveni aliquam abitionem a domino.

Le verb olorizere, qui n'est pas attesté dans la langue latine, est une invention géniale du traducteur à partir du substantif olus / holus (“légumes»); tout aussi ingénieuse est l'utilisation du nom abitio (“ départ »), qui était très rare, pour le grec ámókıvos, que les scholies expliquent par le mot «fugue ${ }^{11}$ ».

Dans le prologue de la comédie il y a d'autres escamotages qui ne peuvent que susciter notre approbation. À partir du vers 40, après son dialogue avec son comparse Nicias, l'esclave Démosthène expose au public la situation en décrivant ainsi son maître:

10 Aristophane, Cavaliers i6, i9-20. La citation d'Euripide vient d'Hippolyte 345.

1 Avec le sens de “départ», abitio est attesté en latin classique seulement dans Plaute, Rudens 5 o3. 


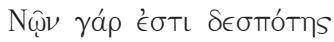

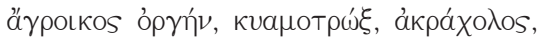

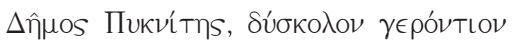

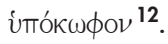

Si nous lisons la traduction de Divus, nous voyons qu'il n'a pas compris que

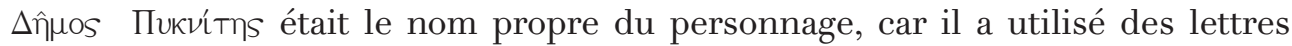
minuscules :

Nobis enim est dominus

rusticus ira, fabarum comestor, promptus in ira,

populus concionarius, difficilis vetulus,

surdulus.

Mais grâce aux scholies il a pu comprendre que Пขкvítᄁs avait un sens métaphorique, et c'est pour cette raison qu'il l'a traduit avec concionarius, qui est un néologisme très efficace.

L'adjective surdulus est aussi une création ingénieuse, car le suffixe -ulus traduit avec succès le préfixe grec iто-.

À cet égard, il faut dire que la fréquence des diminutifs dans la langue flamboyante d'Aristophane a forcé Divus à créer continûment des néologismes. Nous pouvons voir de nombreux cas similaires dans le texte de la comédie: les diminutifs consiliunculis et sententiunculis et cogitatiunculis (on est toujours dans le prologue)

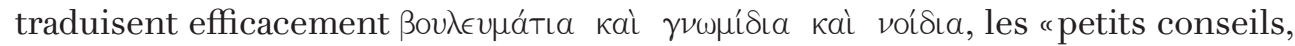
petites sentences, petites idées » que le vin a suggérés aux deux esclaves ${ }^{13}$; les diminutifs urnulis et vulturulis et turriculis (dans les scène iambiques qui suivent la

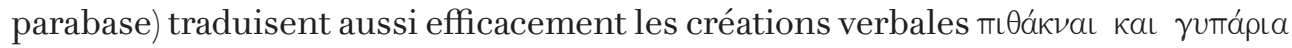

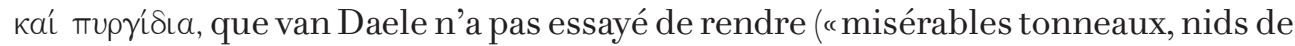
vautours et tourelles s) $)^{14}$.

Si l'on revient au monologue de Démosthène dans le prologue, on remarque une autre création courageuse de Divus: le verbe sybillizare, qui correspond au grec $\sigma \iota \beta u \lambda \lambda_{\iota} \tilde{\nu} \nu$ et décrit la passion du Peuple pour les faux oracles que le Paphlagonien utilise souvent pour tromper son maître ${ }^{15}$.

Or, après avoir volé les oracles du Paphlagonien, les esclaves apprennent qu'il y a une prophétie qui annonce la venue d'un esclave qui prendra la place du Paphlagonien - c'est-à-dire une prophétie annonçant la venue d'un homme politique qui

12 Aristophane, Cavaliers 40-43: “Nous avons un maître rustre d'humeur, croqueur de fèves, prompt à s'irriter, Démos, de Pnyx, petit vieux bourru, à l'oreille dure ».

13 Aristophane, Cavaliers ıoo.

14 Aristophane, Cavaliers 792-793.

15 Aristophane, Cavaliers 6I. 
prendra la place de Cléon. Divus s'efforce de traduire tous les noms composés de

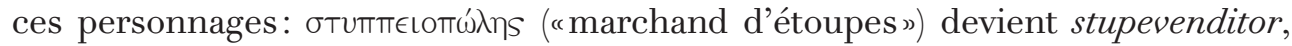

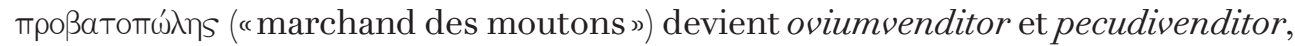

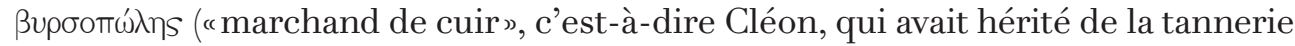
de son père) devient pelliumvenditor ${ }^{16}$. Le seul métier que Divus ne traduit pas, c'est le métier de l'esclave qui viendra prendre la place du Paphlagonien: dans la

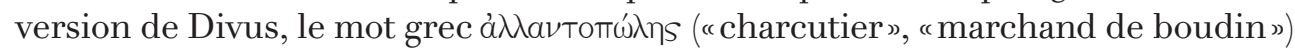
reste toujours allantopoles ${ }^{17}$.

Quand ce charcutier arrive vraiment, les esclaves lui expliquent pourquoi il va devenir un excellent homme politique:

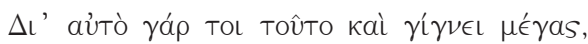

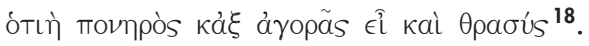

Comme la scholie n'explique pas l'acception particulière du mot áyopá dans ce contexte, Divus ne saisit pas son sens dépréciatif, interprété correctement par van Daele ( Parce que tu es un gueux, un voyou, un audacieux»); par suite, la traduction du deuxième vers (Quod malus, et ex foro es et audax) est incorrecte.

Ce même sens particulier revient dans la liste des qualités du bon démagogue que Démosthène dresse peu après :

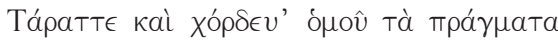

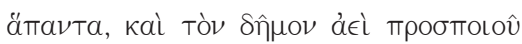

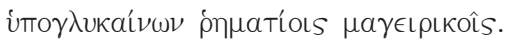

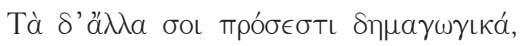

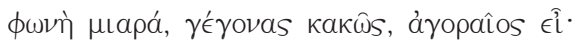

ËX

Voici la traduction de Divus :

Turba, et inchorda simul res

omnes, et populum semper vendica,

subdulce capiens verbulis coquinariis.

Haec autem alia tibi adsunt populi ductiva, vox scelesta, genitus es malus, forensis es.

Habes omnia illa ad civilitatem quae oportent.

16 Aristophane, Cavaliers i29, I36, I38 et I39.

17 Aristophane, Cavaliers 143.

18 Aristophane, Cavaliers I80-I8I.

19 Aristophane, Cavaliers 2I4-2I9. 
Son forensis n'a pas le sens correct que lui donne van Daele (“façons de voyou»).

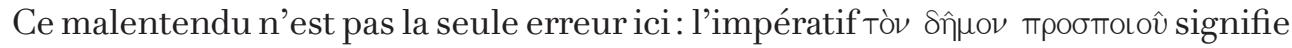
"gagne toujours pour toi la faveur du peuple»; le dernier vers signifie "tu as tout ce qu'il faut pour gouverner». Mais il y a aussi des solutions intéressantes: avec subdulce capiens, Divus renonce pour une fois à la coutume qui l'emmène toujours vers une traduction littéraire, en paraphrasant correctement le verbe composé

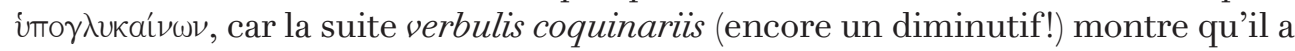
compris et respecté la métaphore culinaire d'Aristophane.

Les esclaves encouragent le charcutier à lutter contre le Paphlagonien en l'assurant de l'aide des Cavaliers; le Paphlagonien arrive furieux et menaçant; Démosthène appelle les Cavaliers, qui frappent le démagogue. Dans la dernière section de la parodos, nous trouvons une première altercation entre le Charcutier, qui est enhardi par la présence du chœur des Cavaliers, et son adversaire. Divus essaie de reproduire les figures étymologiques du texte grec: par declamabo

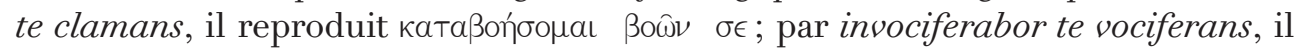

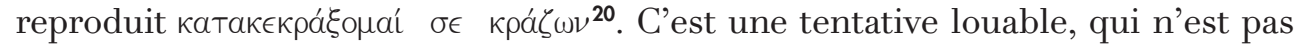
fréquente chez les traducteurs. Van Daele, par exemple, ne le fait pas, car il traduit: “Je couvrirai ta voix par mes cris » et «Moi la tienne par mes beuglements ».

Pendant le débat, le Paphlagonien est battu soit en paroles soit en action. Il compte prendre sa revanche devant le Conseil, mais après la parabase le Charcutier annonce qu'il a été vainqueur devant le Conseil aussi. Dans son compte-rendu du discours du Paphlagonien, il décrit ainsi l'éloquence de son rival:

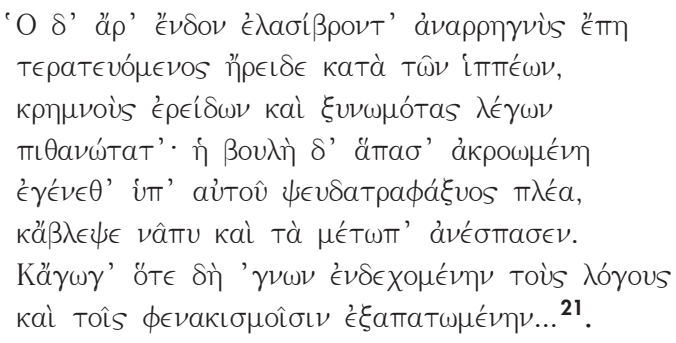

La densité métaphorique du premier vers est trop difficile pour Divus: la

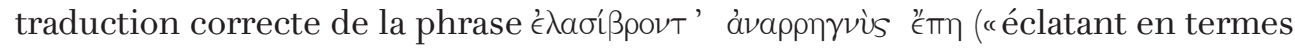
tonitruants ", selon van Daele) devient scindens impellentia tonitrua verba; mais les

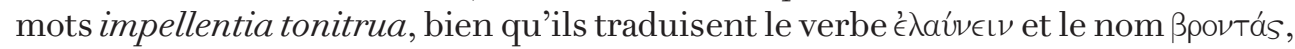
ne signifient pas grand chose.

20 Aristophane, Cavaliers 286-287.

21 Aristophane, Cavaliers 626-633. 
Le participe qui suit, mentiens, est toutefois correct: le verbe utilisé par Divus est la vraie traduction du grec $\tau \epsilon \rho a \tau \epsilon \cup o ́ \mu \epsilon \nu o s$, parce que tépas ne signifie pas seulement "prodige», mais aussi “mensonge» - et Divus, qui avait lu la scholie, qui explique que le verbe est un synonyme de $\psi \in \cup \delta o ́ \mu \epsilon \nu o s$, a choisi le participe latin correct.

Et c'est toujours grâce aux anciens commentateurs que Divus a pu comprendre

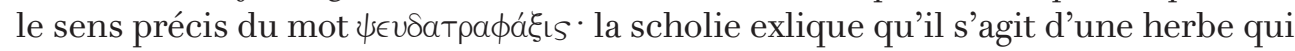
pousse rapidement; Divus ne prête pas attention à l'image végétale mais, s'appuyant sur le premier élément du composé, traduit correctement le mot par levia mendacia.

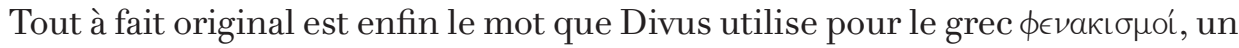
terme qui dérive du mot $\phi \epsilon ́ v a \xi$ («perruque»): hallucinationes est une traduction singulièrement métaphorique pour un traducteur qui est d'ordinaire assez fidèle.

Mais le Paphlagonien ne se tient pas pour définitivement battu. Il veut soutenir maintenant un débat devant Peuple; le Charcutier y consent.

Peuple arrive. Le Charcutier l'appelle avec le vocatif $\hat{\omega}^{\prime} \Delta \eta \mu_{i} \delta\llcorner\mathrm{\nu}$, rendu par le diminutif popellus, un terme que Divus a emprunté à une épître d'Horace - une autre preuve qui démontre qu'il connaissait bien les auteurs latins ${ }^{22}$. Ensuite, les deux démagogues font assaut de flatteries, de complaisances et de cadeaux.

Regardons le passage où le chœur des Cavaliers blâme l'ignorance et la corruption du Paphlagonien, ici appelé par son vrai nom pour la première fois :

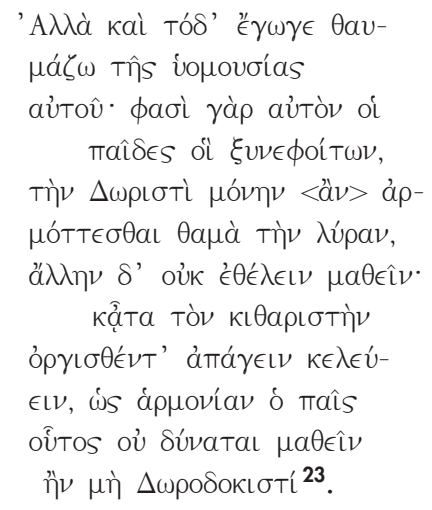

Ce passage pose des problèmes à tous les traducteurs, parce qu'Aristophane y

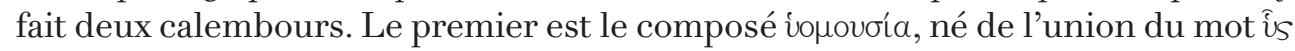

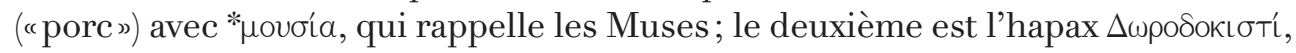

22 Aristophane, Cavaliers 726; Horace, Epistulae I, 7, 65.

23 Aristophane, Cavaliers 984-996. 
un adverbe qui joue soit sur le mode dorien, un des quatre modes de la musique grecque, soit sur Doro, qui était la déesse de la vénalité, dont le nom renvoie à

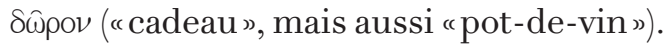

Voici la traduction de Hilaire van Daele:

«Mais ce trait encore, je l'admire dans son éducation de pourceau :

les enfants qui fréquentaient l'école avec lui

disent qu'il lui arrivait souvent de ne pouvoir accorder sa lyre

que sur le mode dorique et qu'il se refusait à en apprendre un autre;

alors le maître se fâchant le faisait emmener,

attendu, disait-il, que cet enfant n'est capable d'apprendre aucun mode,

si ce n'est le $<$ louis d'or $>$ ique».

Et Divus? Sa traduction est la suivante:

Sed et hoc ego ad-

miror scientiae porcinae

ipsius. Dicunt enim ipsum

pueri qui simul ibant,

donative solam co-

aptare frequenter lyram,

aliam autem non velle discere:

et postea citharistam

iratum abducere iube-

re, quod harmoniam puer

hic non potest discere,

nisi donorum susceptive.

En ce qui concerne le premier calembour, on voit que la scientia porcina de Divus est une traduction très amusante du composé grec, peut-être moins banale et plus comique que la solution “éducation de pourceau » proposée par van Daele.

Le deuxième calembour est traduit avec une imagination appréciable: pour

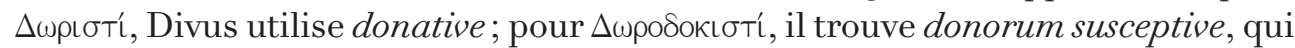
me paraît une solution moins alambiquée que «le $<$ louis d'or $>$ ique » de van Daele.

La fin de ce deuxième débat voit la victoire définitive du Charcutier; Peuple le nomme son intendant à la place du Paphlagonien disgracié, avec l'approbation enthousiaste du chœur des Cavaliers. À la fin de la comédie, rajeuni par un procédé magique, Peuple abjure ses erreurs passées et promet de mieux faire à l'avenir.

Parmi les premières mesures du Peuple, auxquelles le Charcutier donne son approbation, il y a l'interdiction faite aux «freluquets du marché au parfums » (Tà

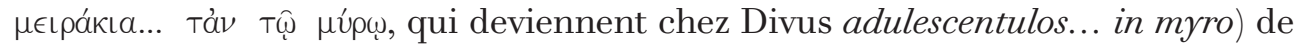
flâner sur l'agora; ainsi, ils ne pourront plus parler avec leur langage ridiculement 


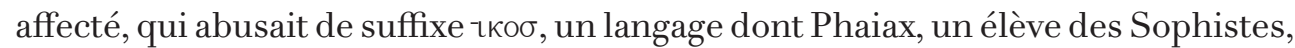
était un des inventeurs.

Dans la définition que Peuple donne de Phaiax on aperçoit tout le sarcasme d'Aristophane sur cette façon ridicule de parler:

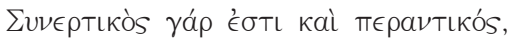

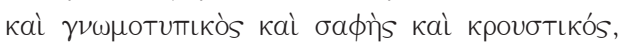

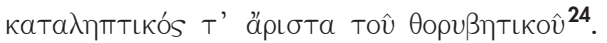

Divus comprend l'ironie d'Aristophane et essaie de la reproduire en utilisant le suffixe latin -ivus:

Compositivus enim est et terminativus,

Et sententiarum formativus, et manifestus, et pulsativus,

Deprehensivusque optime tumultuarii.

Le résultat est assez satisfaisant - au début, au moins, parce qu'à la fin Divus

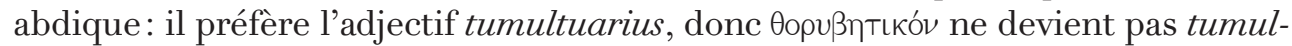

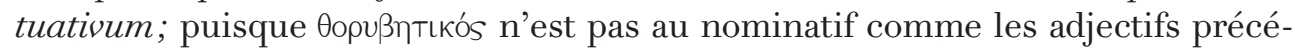
dents, Divus a peut-être cru qu'il ne concernait pas le sophiste ${ }^{25}$.

Enfin, Peuple condamne le Paphlagonien à revêtir le costume du Charcutier et à prendre le métier du marchand qui l'a supplanté dans sa position politique: il faudra qu'il vende les saucisses aux portes de la ville (et, en sus, qu'il se querelle avec des prostituées et des baigneurs). La dernière définition du Paphlagonien vaincu est le mot фapнакós. Pour van Daele, le sens du mot est «malfaiteur»; pour Divus, c'est magus.

Mais que signifie vraiment ce mot? Les scholies l'ignorent. En latin, dans un contexte géographique particulier, celui de la Perse, magus signifie "prêtre» ou “devin ${ }^{26}$ »; dans d'autres contextes, il veut dire simplement “ magicien » ${ }^{27}$. Ni “prêtre» ni «devin» ni «magicien» n’ont quoi que ce soit à voir avec la situation; Divus a probablement essayé lui-même d'être un magus, c'est-à-dire de deviner le sens du mot - une chose qu'il a dû faire souvent, soit dans les comédies

24 Aristophane, Cavaliers i378-ı38o.

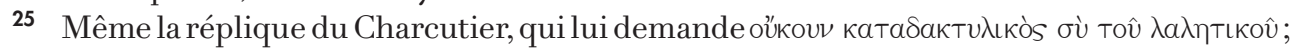
(v. I38I: “N'es-tu pas l'habile manipulateur de cet habile phraseur?»), est traduite par Divus sans recourir au suffixe -ivus (Igitur contra annulum illum loquacem?).

26 Cicéron, Leges 2.26.

27 Horace, Carmina I.27.22; Ovide, Métamorphoses 7.195. 
d'Aristophane soit dans la plus connue de ses traductions, celle des deux poèmes d'Homère ${ }^{28}$. Est-ce qu'il faut le blâmer pour ça?

Malgré tous ses défauts, il faut toutefois reconnaître que Divus a essayé de se battre «avec le courage d'Héraclès » contre le «monstre» Aristophane ${ }^{29}$ : il l'a suivi dans ses nombreuses créations linguistiques (en créant à son tour de nouveaux mots latins); il l'a suivi dans ses altérations linguistiques (en altérant à son tour plusieurs mots, c'est-à-dire en créant des diminutifs); il l'a suivi dans ses calembours fantaisistes (en essayant parfois de les reproduire avec une fantaisie à peu près équivalente).

Si ses tentatives ne sont pas toujours réussies et si sa connaissance de la langue grecque laisse parfois à désirer, on ne peut pas nier que, sans la traduction de Andreas Divus, la connaissance du théâtre d'Aristophane se serait répandue beaucoup plus lentement. Surtout, s'il n'avait pas traduit toutes les onze comédies conservées par les manuscrits médiévaux, nous n'aurions pas pu lire à la même période la première traduction complète du théâtre d'Aristophane dans une langue moderne: à savoir la traduction de frères Pietro et Bartolomeo Rositini, publiée à Venise en 1545 , sept ans après la traduction latine de Divus ${ }^{30}$.

Cette traduction italienne se base très souvent (et même trop souvent: même une lecture superficielle permet de constater que bien des bévues de Divus sont passées presque intactes dans la traduction des frères Rositini) sur la première

28 Homeri Ilias, Andrea Divo Iustinopolitano interprete, ad verbum translata. Herodoti Halicarnassei libellus, Homeri vitam fidelissime continens, Conrado Heresbachio interprete. Cum indice copiosissimo, Venetiis, apud D. Iacob a Burgofrancho Papiensem, mense Martio, I537; Homeri... Odyssea, Andrea Divo Iustinopolitano interprete, ad verbum translata. Eiusdem Batrachomyomachia, id est, Ranarum et murium pugna, Aldo Manutio Romano interprete. Eiusdem Hymni deorum 32. Georgio Dartona Cretense interprete, Venetiis, apud D. Iacob a Burgofrancho Papiensem, mense Martio, I537. Divus traduisit aussi les Idylles de Théocrite (Theocriti Syracusani poetae clarissimi Idyllia trigintasex, recens e graeco in latinum, ad verbum translata, Andrea Divo Iustinopolitano interprete. Eiusdem Epigrammata, Bipennis, Ala, \& Ara, latinitati donata, eodem Andrea Divo interprete, Venetiis, apud D. Iacob a Burgofrancho Papiensem, mense Februario, I539).

29 J'ai emprunté une image que le même Aristophane a utilisée deux fois pour décrire sa lutte contre le «monstre "Cléon (Guêpes Io29-ı037 et Paix 752-76o).

30 Sur cette traduction, voir S. BEtA, “Aristophanes Venetus : i fratelli Rositini e la prima traduzione italiana del poeta comico greco (I545)», Cahiers d'études italiennes I7 (2013), p.57-7o; sur les traductions italiennes en général, voir R. QuagLIA, “Su alcune traduzioni italiane di Aristofane: azzeccagarbugliando tra i secc. xVI e xIX », Maia 57 (2006), p.349-357. 
traduction latine intégrale ${ }^{31}$. Mais Pietro et Bartolomeo, qui n'étaient pas des professionnels - ils exerçaient la profession de médecin - ont parfois montré une autonomie de jugement.

Par exemple, ils ont traduit le фapнaкós dont je viens de parler par «incantatore », qui veut dire surtout «enchanteur», mais qui signifie aussi «charmeur », un mot qui apparemment ne convient pas pour désigner un démagogue vaincu qui est destiné, comme le dit Aristophane, à injurier les prostituées et à boire l'eau sale des bains (ou, si l'on veut utiliser les mots de Divus, meretricibus conviciari et ex balneis ipsum lavacrum bibere $)^{\mathbf{3 2}}$.

Mais on sait bien que les vrais démagogues, même s'ils ont été vaincus, même s'il semble qu'ils sont en train de quitter leur charge, sont toujours capables de se cramponner à toutes les qualités qu'ils possèdent - y compris la qualité des $\phi \alpha \rho \mu \alpha^{-}$ кó - au sens de «incantatori », “enchanteurs », mais aussi “charmeurs ». Par suite, comme nous savons que, après avoir voté pour la victoire d'Aristophane dans la compétition théâtrale des Lénéennes, les Athéniens votèrent pour l'élection de Cléon, qui cependant avait été tourné en ridicule dans une comédie qui lui était entièrement consacrée et qui fut acclamée par le public, la traduction des frères Rositini n'est pas complètement incorrecte.

La traduction donnée par Nicodemus Frischlin en i586 est plus juste, bien sûr, car son veneficus reproduit fidèlement le ton dépréciatif du terme grec ${ }^{33}$. Mais les deux mots choisis quelques années avant par Divus et par les frères Rositini possèdent un mérite, bien que ce mérite soit involontaire: ils sont une définition inconsciente de leur travail de traducteurs.

Un homme qui traduit est un incantatore, parce qu'il doit reproduire dans sa propre langue le même charme que les mots utilisés par l'auteur avaient dans la langue originale. Mais un traducteur est un magus aussi : ils doit « deviner» le sens correct des mots qu'il trouve dans le texte qu'il doit traduire et, comme par enchantement, le transporter dans sa langue. Face au texte des comédies d'Aristophane, écrit dans une langue qui était encore peu connue, Andreas Divus a essayé d'être

31 Sur les rapport entre la traduction de Divus et celle des Rositini, voir aussi F. SchreIber, "The Etiology of a Misinterpretation: Aristophanes Birds 3o , Classical Philology 70 (I975), p. 208-2I2.

32 Aristophane, Cavaliers $1400-$-140I.

33 Nicodemi Frischlini Aristophanes, veteris comoediae princeps: poeta longe facetissimus \& eloquentissimus: repurgatus a mendis, et imitatione Plautiatque Terentii interpretatus, ita utfere carmen carmini, numerus numero, pes pedi, modus modo, Latinismus Graecismo respondeat, Francoforti ad Moenum, apud Iohannem Spies, i586. Frischlin traduisit seulement cinq comédies (Ploutos, Nuées, Grenouilles, Cavaliers et Acharniens); ses traductions latines furent utilisées par Ludolf Küster dans l'édition intégrale des comédies d'Aristophane qu'il publia en Ijıo (voir n. 6). 
un magus, car il a tenté de transformer un poète ayant vécu dans l'Athènes démocratique du $v^{e}$ siècle avant J.-C. en un auteur s'exprimant dans la langue franche de l'Europe occidentale. Cette magie n'a pas toujours été couronnée de succès, c'est vrai - mais le magus Divus mérite malgré tout nos bravos.

\section{Simone Beta}

Dipartimento di Filologia e Critica delle Letterature Antiche e Moderne Università di Siena

Via Roma 56

53100 Siena

beta@unisi.it 